\title{
Refined electron-spin transport model for single-element ferromagnetic systems: Application to nickel nanocontacts
}

\author{
W. Dednam $\odot,{ }^{1,2, *}$ C. Sabater $\odot,{ }^{3}$ O. Tal $\odot,{ }^{3}$ J. J. Palacios $\odot,{ }^{4}$ A. E. Botha $\odot,{ }^{2}$ and M. J. Caturla $\oplus^{1}$ \\ ${ }^{1}$ Departamento de Física Aplicada and Unidad Asociada CSIC, Universidad de Alicante, Campus de San Vicente del Raspeig, \\ E-03690 Alicante, Spain \\ ${ }^{2}$ Department of Physics, Unisa Science Campus, University of South Africa, Johannesburg 1710, South Africa \\ ${ }^{3}$ Chemical Physics Department, Weizmann Institute of Science, 76100 Rehovot, Israel \\ ${ }^{4}$ Departamento de Física de la Materia Condensada, Condensed Matter Physics Center (IFIMAC) and Instituto Nicolás Cabrera, \\ Universidad Autónoma de Madrid, Madrid E-28049, Spain
}

(Received 5 September 2020; revised 8 November 2020; accepted 16 November 2020; published 14 December 2020)

\begin{abstract}
Through a combination of atomistic spin-lattice dynamics simulations and relativistic $a b$ initio calculations of electronic transport we shed light on unexplained electrical measurements in nickel nanocontacts created by break junction experiments under cryogenic conditions $(4.2 \mathrm{~K})$. We implement post-self-consistent-field corrections in the conductance calculations to account for spin-orbit coupling and the noncollinearity of the spins, resulting from the spin-lattice dynamics. We find that transverse magnetic domain walls are formed preferentially in (111)-oriented face-centered-cubic nickel atomic-sized contacts, which also form elongated constrictions, giving rise to enhanced individual domain wall magnetoresistance. Our calculations show that the ambiguity surrounding the conductance of a priori uniformly magnetized nickel nanocontacts can be traced back to the crystallographic orientation of the nanocontacts, rather than spontaneously formed magnetic domain walls "pinned" at their narrowest points.
\end{abstract}

DOI: 10.1103/PhysRevB.102.245415

\section{INTRODUCTION}

The modeling of dynamic and thermal effects on spins in ferromagnetic systems is a challenging problem [1,2], since the inclusion of spin-orbit coupling (SOC) becomes essential at the atomic scale [3-5]. If, furthermore, the goal is to account for electronic transport in these systems, the task becomes even more complicated. Over the past few years, a number of implementations for quantum transport based on density functional theory (DFT) have appeared in the literature [6-21], although modeling complex ferromagnetic systems (beyond atomic linear chains and toy model contacts) is still computationally challenging. Requist et al. [22], for example, have recently compiled a detailed summary of the state of the art in electronic transport calculations on nickel nanocontacts.

Our present implementation of the quantum transport calculation addresses several important effects, simultaneously. It consists of an atomistic multiphysics approach that combines spin-lattice dynamics (SLD) and DFT electronic transport calculations in a unique way. We include the magnetic anisotropy corrections in our SLD calculations in order to be able to model accurately the magnetoelastic effects in low-dimensional ferromagnetic structures [4,5]. Combined with the SLD model, we use a post-self-consistent-field $a b$ initio methodology to calculate electronic transport that takes

\footnotetext{
*wd2@alu.ua.es
}

into account the effect of the SOC $[21,23]$ as well as the contribution from noncollinearity (NC) in the spins [24,25].

While our combined model can conceivably be applied to several, low-dimensional, magnetic systems, in order to elucidate the exact roles played by the previously described effects, here we exemplify its use by applying it to the low-temperature experiments of electronic transport in atomic-sized contacts made of pure nickel [26-30]. We have specifically selected ferromagnetic nickel because the interpretation of its experimentally measured conductance histograms remains enigmatic, as we explain next.

Typically, electron transport measurements on nanocontacts are expressed as the conductance vs the relative displacement between the atomic-sized electrodes. The most widely used statistical analysis of these measurements is the histogram of conductance, which shows the distribution of the conductance values measured repeatedly for the same junction. Usually, the histogram is like a "fingerprint" of the metal, i.e., it characterizes the metal uniquely [31]. However, in the case of nickel nanocontacts, two distinct histograms have been measured [26-30]. The most frequent histogram recorded for $\mathrm{Ni}$ over the years exhibits a single broad first peak, denoted the single peak (or "1-peak") histogram in this work [28-30,32]. On the other hand, a "2-peak" histogram has also been obtained which exhibits two narrower peaks in place of the broad peak of the 1-peak histogram [26,27]. Until now, this ambivalence in the measurements could not be explained by previous models.

Different research groups have attempted to explain the two distinct experimental histograms of Ni. Garcia-Mochales 
et al. [33] used classical molecular dynamics (CMD) simulations to generate minimum cross-section histograms of $\mathrm{Ni}$ nanowires oriented along the (001), (111), and (110) crystallographic axes. They did not perform electronic transport calculations, but speculated that the two distinct histograms might be explained by the formation, on one hand, of a dimeric last contact (a two-atom chain suspended between the bulk electrode tips immediately before rupture), where a less frequent conductance peak at a low value of $\sim 1.2 G_{0}$ is observed in experiments, and, on the other, a monomeric last contact (a single atom bridging the two electrodes) at the most frequent last contact value of $\sim 1.6 G_{0}$ measured in experiments. (The quantum unit of conductance $G_{0}$ is defined as $G_{0}=2 e^{2} / h$, where $e$ is the electron's charge, $h$ is the Planck's constant, and the factor 2 comes from spin degeneracy.) In the same year, Calvo et al. [26] attempted to explain the two distinct histograms they reproduced experimentally by following the same statistical approach as Garcia-Mochales et al. [33] in their CMD simulations, but, in addition, performing DFT electronic transport calculations on small Ni toy model contacts. The electronic transport calculations were inconclusive and they reported the most frequent value of $\sim 1.6 G_{0}$ for both dimeric and monomeric last contact structures, even after stretching and relaxing these structures in their DFT calculations. Based on this finding, Calvo et al. speculated that the lower peak of conductance obtained less frequently in experiments might instead be explained by magnetoresistive effects caused by the presence of magnetic domain walls pinned at the constriction. Vardimon et al. [28] constructed a theoretical conductance histogram from electronic transport calculations on atomic structures obtained from 100 ensemble CMD rupture simulations. Their input model structure was oriented along the (100) crystallographic axis and they obtained a most frequent last contact conductance value of $\sim 1.6 G_{0}$.

In this work we use our combined methodology to untangle some of the physics leading to the observed differences between the two types of experimental histograms for nickel nanocontacts. However, we would like to stress that, because of the prevalence of emergent magnetic phenomena within a wide variety of systems, we envisage that the same method may also be of a wider use. For example, it could be applied in studies of skyrmions [2,34], magnetic nanowires [35], and magnetic nanoparticles [36], as well as to various well-known effects, such as, Einstein-de Haas [37], Barnett [38], and Barkhausen [39].

\section{THEORETICAL AND EXPERIMENTAL APPROACH}

In Secs. II A-II C, we briefly describe the two complementary parts of our methodology, with specific emphasis on on the modifications we made in comparison to previous implementations, and our motivation for making these modifications. In Sec. II D we discuss how the electronic transport experiments on Ni nanocontacts were performed.

\section{A. Improved spin-lattice dynamics model}

The first part of our methodology builds on the technique known as spin-lattice dynamics (SLD) [40-42]. This is a semiclassical molecular dynamics approach which allows one to evolve simultaneously the atomic spin and translational degrees of freedom. The interatomic interactions in SLD are described by the widely used embedded atom method (EAM) potential [43]. Previously, it has been implemented in codes such as SPILADY along with a generalized Heisenberg model of (local interatomic) ferromagnetic exchange [40,42]. The specific EAM potential we use for $\mathrm{Ni}$ was developed in Ref. [44]. In particular, it has, among many bulk properties, been fitted to at least one surface property: the heat of sublimation of the metal [45].

As in our previous works and following the experimental conditions [46-48], we maintain the temperature of the atoms in our simulations at $4.2 \mathrm{~K}$, i.e., the temperature of liquid helium. We employ a Langevin thermostat for this purpose, as described in Refs. $[3,4,49]$. Here, we introduce also the evolution of the spins, keeping them at the same temperature.

To emulate the controlled rupture and formation of the contacts in the experiments, i.e., the process of cyclic loading, we have implemented the algorithm described in Refs. [50-52] in the SPILADY code. In our SLD simulations, the atoms and spins in the two outermost layers on each side of the contact are frozen and displaced at $\sim 1 \mathrm{~m} / \mathrm{s}$ in opposite directions. The atoms and spins sandwiched between the frozen layers are then allowed to evolve dynamically in response to the displacements, as the simulation proceeds.

The generalized Heisenberg ferromagnetic exchange model used to describe spin-spin and spin-lattice interactions in SPILADY has two fitting parameters [40,42]: an exchange coefficient $J_{0}$ and a pairwise cutoff distance $r_{c}$. For the Ni in this work, we use $J_{0}=0.83 \mathrm{eV}$ and $r_{c}=4.34 \AA$, which have been obtained from unpublished data [53], by a similar fitting procedure to that reported in Ref. [54]. The chosen value of $r_{c}$ effectively cuts off the exchange interaction between second and third nearest neighbors.

The above model of ferromagnetic exchange is isotropic and hence does not conserve the angular momentum of the combined lattice and spin system $[4,5]$. Furthermore, it is well known that magnetic anisotropy, whose origin can be traced to SOC, cannot be ignored in low-dimensional systems such as ferromagnetic nancontacts $[19,55]$. For this reason we have added the second-order (uniaxial) anisotropy correction, described in Ref. [4], to the SPILADY code. We have omitted the first-order anisotropy correction simply because it does not preserve time-reversal symmetry.

The second-order anisotropy correction introduces two new parameters into our model, namely, the coefficient $C_{2}$ and the new cutoff distance $r_{0}$, for an EAM electron-density-like function that accounts for the asymmetric environment of the spin vector centered on a given atom. As discussed in Ref. [4], there is currently no accurate way to fit these two parameters, experimentally or otherwise. In fact, even the coefficient of the simpler anisotropy correction used in Ref. [5] exhibits uncertainty of up to an order of magnitude compared to the available experimental results. Consequently, in analogy with Ref. [4], we choose a slightly smaller cutoff of $r_{0}=3.75 \AA$ than is used for $r_{c}$ in the isotropic exchange term. By performing several trial simulations we found that, as the value of $C_{2}$ decreases and becomes negative, spin alignment perpendicular to the length of the nanocontact becomes more prevalent. With the 
objective of ascertaining the effects of transverse domain wall formation at the constrictions of the nanocontacts, we have chosen $C_{2}=-0.5 \mathrm{eV}$ in our present simulations.

We emphasize that our SLD model rigorously conserves total energy and angular momentum [5], and encourage the interested reader to consult Ref. [52] for an in-depth discussion of its implementation as well as several benchmark calculations.

\section{B. Post-self-consistent-field SOC corrections to DFT electronic transport calculations}

The spin-lattice dynamics model mentioned in Sec. II A provides more realistic coupled atomic and spin configurations. Often, the experimental characterization of nanostructures is achieved by measuring their electronic properties. This is the case, for example, of conductance traces obtained via STM for nanocontacts near the moment they are about to rupture $[47,56]$. In order to make comparisons with such experimental observations, the electronic transport of these structures must be accurately modeled.

The code ALICANTE NANOTRANSPORT (ANT.GAUSSIAN) [57-60] has been used successfully in the past to perform conductance calculations on CMD simulation snapshots near rupture [46-48]. This code interfaces with GAUSSIAN09 [61] to perform a scalar-relativistic, spin-unrestricted calculation of the transport, i.e., it assumes the magnetism is collinear.

Spin-orbit coupling, which is considered to be an intraatomic phenomenon, and noncollinear spin configurations are the obvious missing ingredients in the aforementioned calculations. The self-consistent-field implementation of SOC and noncollinear (NC) magnetism in density functional theory (DFT) codes such as QUANTUM ESPRESSO [62] and OPENMX [18], aside from being extremely expensive computationally, lead to calculations in which there are many local minima, making it more difficult to achieve convergence $[22,28]$. It would be thus extremely difficult, if not impossible, to obtain converged noncollinear spin solutions using only atomic structures from ordinary classical molecular dynamics simulations in such DFT calculations. Therefore, in this work, we make use of a post-self-consistent-field approach as described in Ref. [23] and the references therein. This approach is valid for materials such as $\mathrm{Ni}$ in which SOC is weak [21], but also works quite accurately for materials with strong SOC. We briefly outline it below.

The Dirac-Kohn-Sham Hamiltonian contains to lowest order the standard atomic spin-coupling (SOC) term

$$
\xi(r) \mathbf{L} \cdot \mathbf{S} .
$$

This Hamiltonian is fully relativistic and its electronic and positronic parts are decoupled (see Ref. [23] and references therein). It is evident from the form of this operator that orbital and spin angular momentum are now mixed. Furthermore, as a result of the orthogonality of the radial and angular components of the wave functions in atomic-orbital-based DFT, such as the widely available contracted Gaussian-type orbitals (CGTOs) used by GAUSSIAN09 or CRYSTAL [63], the elements of the SOC matrix can be evaluated simply as [23]

$$
\xi_{i j}\left\langle l_{i} ; m_{l_{i}} ; s|\mathbf{L} \cdot \mathbf{S}| l_{j} ; m_{l_{j}} ; s^{\prime}\right\rangle
$$

where

$$
\xi_{i j}=\frac{e^{2}}{2 m_{e} c^{2}} \int_{0}^{\infty} \frac{1}{r} \frac{d V_{\mathrm{eff}}(r)}{d r} R_{i}(r) R_{j}^{*}(r) r^{2} d r .
$$

In Eq. (2), $V_{\text {eff }}(r)=-\frac{Z}{r}$ is the effective potential in which the electrons move [23], with $Z$ the atomic number. $R_{\mathrm{i}}(r)$ are the radial CGTOs in the GAUSSIAN09 basis sets used in our calculations [23]. Only CGTOs of the same shell type ( $L=1,2$, or 3) contribute to the integral in Eq. (2) for a given atom, to which, in turn, a given basis set has been assigned. The interested reader is referred to Ref. [23] for an in-depth discussion of the above implementation.

\section{Noncollinear spins in DFT electronic transport}

Ferromagnetic nanocontacts are known to exhibit intrinsic magnetoresistance due to domain walls with noncollinear spin structures $[55,64]$. Thus, in order to take into account NC spins, following Refs. [21,24,25,65], the converged (selfconsistent-field) Hamiltonian $\left[H_{\mathrm{loc}}\right]_{\sigma^{\prime} j y}^{\sigma i \mu}$, with majority and minority spins along an arbitrary quantization axis [21,25], is rotated to the orientations of the individual spins with respect to a common quantization axis along the positive $z$ direction:

$$
\left[H_{\mathrm{glob}}\right]_{\sigma^{\prime} j \nu}^{\sigma i \mu}=U(\theta, \phi)\left[H_{\mathrm{loc}}\right]_{\sigma^{\prime} j \nu}^{\sigma i \mu} U^{\dagger}(\theta, \phi) .
$$

The indices $\sigma, \sigma^{\prime}$ refer to "up" or "down" spin-spin combinations [ " $\uparrow \uparrow "$ or " $\downarrow \downarrow$ " in the collinear case on the upper and lower diagonal blocks, respectively, of a doubled-up matrix constructed from the converged (self-consistent-field) Hamiltonian], with each electron spin assumed to be quantized locally along its own individual (unknown) quantization axis [21,25]. The indices $i, j$ refer to different atomic centers, while $\mu, v$ refer to different orbitals and $U(\theta, \phi)$ is a unitary operator that is expressed in terms of Euler angles $(\theta, \phi)$ relative to the global quantization axis:

$$
U(\theta, \phi)=\left(\begin{array}{cc}
e^{-i \frac{\phi}{2}} \cos \left(\frac{\theta}{2}\right) & -e^{-i \frac{\phi}{2}} \sin \left(\frac{\theta}{2}\right) \\
e^{i \frac{\phi}{2}} \sin \left(\frac{\theta}{2}\right) & e^{i \frac{\phi}{2}} \cos \left(\frac{\theta}{2}\right)
\end{array}\right) .
$$

The SOC matrix, which assumes a common quantization along the $z$ axis, is added only after the above rotation has been performed. As an approximation, the hopping as well as interatomic overlap elements between different sites are taken to be the arithmetic average of the respective rotated onsite and intra-atomic overlap elements. The interested reader is referred to Ref. [52] for an in-depth discussion of the subject of this section as well as several benchmark calculations.

\section{Experimental methods: Electronic transport in nickel atomic-sized contacts}

Typically, electron transport measurements on atomicsized contacts are made via scanning tunneling microscopebreak junctions (STM-BJ) [56,66] or mechanically controllable break junctions (MCBJ) $[67,68]$. In this work, the experiments are performed in MCBJ at low temperature $(4.2 \mathrm{~K})$ under ultrahigh vacuum. The sample is prepared from a center-notched $\mathrm{Ni}$ wire $(99.994 \%$ purity, $0.1 \mathrm{~mm}$ diameter, Puratronic supplied by Alfa Aesar) glued to a flexible substrate of phosphor bronze. This substrate receives the percussive impact of the piezoelectric element that acts as firing 


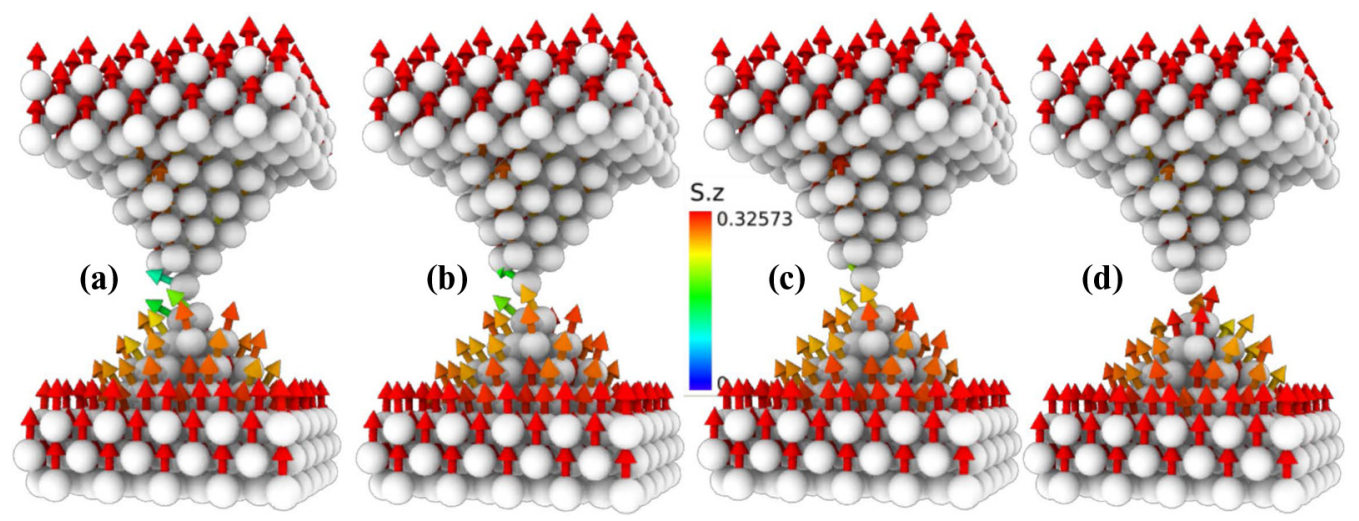

FIG. 1. A 380-atom (001)-oriented Ni nanocontact rupturing at $4.2 \mathrm{~K}$ with a monomer in its minimum cross section (in a sequence of four snapshots). The DWMR for this contact before rupture (a) is $+3 \%$ and corresponds to rupture 10 in Table I. The color legend corresponds to the projection of the spins (in $\mu_{\mathrm{B}}$ ) on the positive $z$ axis, the direction of saturation magnetization of $\mathrm{Ni}, 0.65 \mu_{\mathrm{B}}$, divided by the electron's gyromagnetic factor: 2.002319 .

pin, bending or returning the substrate to its original form. This in turn leads to breaking of the wire. Depending on whether the piezoelectric element is extended or contracted, both segments of the broken wire can reform or break off contact, respectively. In order to measure the electronic transport in the contact, a battery that provides a bias voltage $\left(V_{\text {bias }}\right)$ is connected in series to the nickel contact and an $I-V$ converter amplifier, which, in turn, is connected with our data acquisition and control (DAC) in the computer. Thus, this $I-V$ apparatus can measure the current $(I)$ flowing in the contact. Knowing $I$ and $V_{\text {bias }}$, we can then express the measurement in terms of the conductance, that is, the inverse of the resistance $\left(G=I / V_{\text {bias }}=1 / R\right)$, whose magnitude we express in units of the quantum of conductance $G_{0}$. A measurement of conductance vs the relative piezodisplacement is usually referred to as a trace of conductance, and denoted as a "rupture trace" when the electrodes are pulled apart [67-69]. In the experiments presented here, we applied a bias voltage of 150 $\mathrm{mV}$ and an amplification in the $I-V$ converter of $10^{5}$.

\section{RESULTS}

\section{A. Simulation results}

Through our SLD simulations we have modeled Ni nanocontacts of two different crystallographic orientations along the $z$ axis: (001) and (111) (see Figs. 1 and 2). First, the SLD model is used to stretch the nanocontact until it breaks by displacing the top and bottom layers of the simulation cell, as done previously $[48,70]$. This process is repeated several times in both orientations until the atomic arrangement no longer changes significantly, after so-called mechanical annealing of the tips [51]. The simulation also continues for the same duration in both orientations [71]. The important difference here is that we also follow the evolution of the spins of every atom, as shown in Fig. 1, by the arrows. Subsequently, the electronic transport of these structures is obtained using the implementation described in Secs. II A-II C.

Figure 1 shows a sequence of snapshots of the same atomic and spin configuration, immediately before [Figs. 1(a)-1(c)] and after [Fig. 1(d)] it has ruptured. In this particular case, we performed a total of 13 cycles and the snapshots in Fig. 1 correspond to the 10th rupture cycle. These results are for a 380-atom (001)-oriented $\mathrm{Ni}$ nanocontact at $4.2 \mathrm{~K}$. The last contact in Figs. 1(a)-1(c) is a single atom joining the two tips, that is, a monomer. Note that, despite the fact the parameters for SLD are such that a spin configuration perpendicular to the long axis of the nanocontact is favored, they still remain relatively parallel to the direction of the frozen spins in the bulklike ends of the contact. The relatively flat and wide structure of (001)-oriented tips does not appear to favor the formation of extended transverse domain walls at the constriction immediately before rupture. From the perspective of the spins, this type of contact is disordered, and cooperation among spins within layers perpendicular to the contact's long axis, in order to form transverse domain walls across several layers, is not easy.

Figure 2 shows a sequence of snapshots before [Figs. 2(a)-2(c)] and after [Fig. 2(d)] it has ruptured of a representative atomic and spin configuration of a 417-atom (111)-oriented Ni nanocontact at $4.2 \mathrm{~K}$. In this case, a total of 12 cycles were performed and results in this figure are for rupture number 10 . Note the elongated pentagonal structure that forms during rupture of this (111)-oriented contact, as in Ref. [33]. For simulations of the same duration, there is one fewer rupture cycle in the (111) than (001) orientation because the longer and narrower contacts shown in Fig. 2 take longer to break and make contact again. Clearly, the elongated pentagonal structure of $\mathrm{Ni}(111)$ last contacts allows spins in adjacent layers, perpendicular to the long axis of the nanocontact, to form extended transverse domain walls along this axis. From the perspective of the spins, this contact is more ordered: a domain wall, of atomic scale, has been formed.

As regards electronic transport calculations on our SLD configurations, in order to improve the accuracy of our conductance results, we have assigned an all-electron basis set [72] to 5-10 atoms in the constriction of the nanocontacts extracted from the SLD simulations [48]. We use a minimum $s p d$ basis set for the rest of the atoms [26]. Because we mix basis sets, we have opted to use the literature SOC constants $\xi_{i j}$ in Eq. (1) for Ni: $0.1 \mathrm{eV}$ for the $L=2$ shells [24], and $0.4 \mathrm{eV}$ for the $L=1$ shells (its value should be $3-5$ times that of the $d$ shells), for all basis sets. 


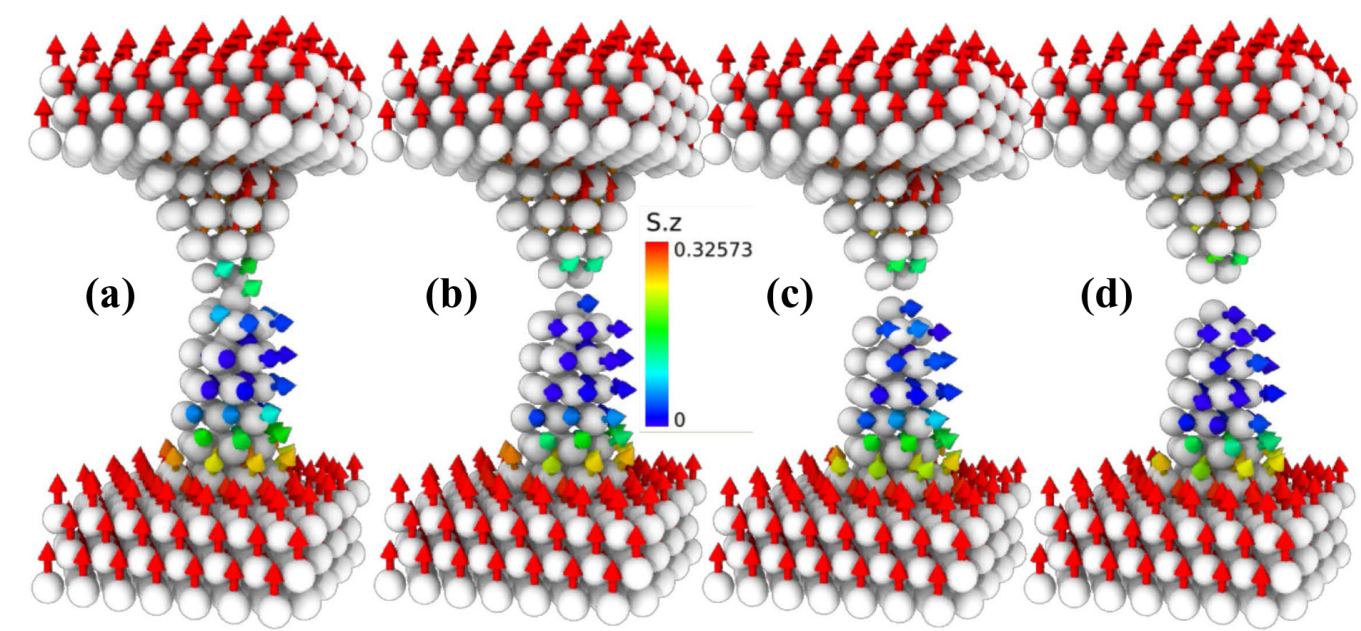

FIG. 2. A 417-atom (111)-oriented Ni nanocontact rupturing at $4.2 \mathrm{~K}$ with a monomer in its minimum cross section (in a sequence of four snapshots). The DWMR for this contact before rupture (b) is $+29 \%$ and corresponds to rupture 10 in Table II. The color legend corresponds to the projection of the spins (in $\mu_{\mathrm{B}}$ ) on the positive $z$ axis, the direction of saturation magnetization of Ni, $0.65 \mu_{\mathrm{B}}$, divided by the electron's gyromagnetic factor: 2.002319 .

To account for the effect on transport calculations of the spin orientations in the SLD snapshot configurations, we have performed both collinear and noncollinear electronic transport calculations with SOC corrections. The spins of bulk $\mathrm{Ni}$ are expected to remain largely collinear at low temperatures [73]. However, this is not necessarily the case in very low-dimensional structures such as nanocontacts $[55,64]$. In this work, the domain-wall magnetoresistance (DWMR) is defined as $\left(G_{\mathrm{NC}}-G_{\text {sat }}\right) / G_{\text {sat }} \times 100 \%$, where $G_{\mathrm{NC}}$ is the SOCcorrected conductance of a SLD snapshot with a noncollinear spin configuration, and $G_{\text {sat }}$ (also SOC corrected) is that of the same snapshot with collinear spins aligned along the positive $z$ axis, i.e., fully saturated. The NC textures ( $\theta$ and $\phi$ angles) are extracted directly from our SLD simulations, while in the collinear calculations all the spins are assumed to be quantized along the global $z$ axis, which also coincides with the transport direction. We emphasize again that, without our SLD simulations, it would be a near impossible task to obtain converged $\mathrm{NC}$ spin textures in fully relativistic DFT calculations on input structures such as those modeled in this work.

Furthermore, to obtain statistically significant results within a reasonable time, we have trimmed down the $(\sim 400$ atom) SLD snapshots to between 100 and 150 atoms. The reduced structures remain centered on the constriction, however. Each snapshot was extracted prior to the moment of rupture, judged to have occurred when the last two atoms to lose contact were separated by a distance equal to halfway between first and second nearest neighbors [74] in a perfect face-centered-cubic (fcc) lattice of $\mathrm{Ni}$, or $\sim 3.0 \AA$.

Tables I and II show the conductance and DWMR results obtained at the last contact for each of the rupture cycles for the (001) and (111) structures. Column type refers to the configuration at the narrowest part of the constriction in terms of number of nearest neighbors [47]. For example, 3-1-1-4 has three atoms in one tip connected to a single one which is also connected to a second single atom and this one, in turn, to four atoms in the other tip. Therefore, this configuration will represent a dimer. A monomer corresponds to types such as 2-1-2 and similar. The calculated DWMR of the monomer in Fig. 1(a) is $+3 \%$ (see rupture 10 in Table I) while for the snapshot in Fig. 2(b), it is $+29 \%$ (see rupture 10 in Table II). Clearly, the presence of a transverse domain wall has a non-negligible impact on individual conductance values, and this is reflected in the values reported in Tables I and II, where conductance is either reduced or increased when transverse domain walls are formed, giving rise to enhanced but either positive or negative DWMR values. The DWMR is greatest when the spins are perpendicular to the transport direction as in Fig. 2. Interestingly, with domain walls the conductance can sometimes be higher than when the spins are collinear [this happens in particular for (001)-oriented nanocontacts and monomer configurations]. We attribute this to the effect of SOC which, as shown in Ref. [21], seems to enhance the conductance for spins oriented perpendicularly to

TABLE I. Contact type, conductance $\left(G_{0}\right)$ with collinear (saturated) and noncollinear SOC, and domain-wall magnetoresistance (\%) of last atomic contact of rupture in simulation with a (001)oriented nanocontact.

\begin{tabular}{lcccr}
\hline \hline Rupture & Type & $\begin{array}{c}\text { SOC-sat } \\
\left(G_{0}\right)\end{array}$ & $\begin{array}{c}\text { SOC-NC } \\
\left(G_{0}\right)\end{array}$ & $\begin{array}{r}\text { DWMR } \\
(\%)\end{array}$ \\
\hline 1 & $3-1-1-4$ & 1.19 & 1.27 & 6.72 \\
2 & $2-1-1-4$ & 1.65 & 1.62 & -1.81 \\
3 & $2-1-3$ & 1.77 & 1.79 & 1.13 \\
4 & $4-2-6$ & 2.50 & 2.53 & 1.20 \\
5 & $2-1-1-4$ & 1.72 & 1.51 & -12.21 \\
6 & $4-2-2-5$ & 2.20 & 2.0 & -9.09 \\
7 & $2-1-4$ & 1.97 & 1.99 & 1.02 \\
8 & $2-1-4$ & 2.00 & 2.05 & 2.50 \\
9 & $2-1-4$ & 1.83 & 1.86 & 1.64 \\
10 & $2-1-4$ & 1.86 & 1.92 & 3.23 \\
11 & $2-1-4$ & 1.99 & 2.02 & 1.51 \\
12 & $2-1-4$ & 1.97 & 1.99 & 1.02 \\
13 & $2-1-4$ & 1.94 & 1.98 & 2.06 \\
\hline \hline
\end{tabular}


TABLE II. Contact type, conductance $\left(G_{0}\right)$ with collinear (saturated) and noncollinear SOC, and domain-wall magnetoresistance (\%) of last atomic contact of rupture in simulation with a (111)oriented nanocontact.

\begin{tabular}{lcccr}
\hline \hline Rupture & Type & $\begin{array}{c}\text { SOC-sat } \\
\left(G_{0}\right)\end{array}$ & $\begin{array}{c}\text { SOC-NC } \\
\left(G_{0}\right)\end{array}$ & $\begin{array}{r}\text { DWMR } \\
(\%)\end{array}$ \\
\hline 1 & $2-1-2$ & 1.55 & 1.56 & 0.64 \\
2 & $2-1-1-3$ & 0.92 & 0.89 & -3.26 \\
3 & $2-1-1-3$ & 1.24 & 1.09 & -12.10 \\
4 & $2-1-1-3$ & 1.09 & 1.11 & 1.83 \\
5 & $2-1-1-5$ & 1.36 & 1.24 & -8.82 \\
6 & $2-1-1-3$ & 0.90 & 0.89 & -1.11 \\
7 & $2-1-5$ & 1.36 & 1.41 & 3.68 \\
8 & $2-1-5$ & 1.59 & 1.32 & -16.98 \\
9 & $2-1-5$ & 1.60 & 1.79 & 11.88 \\
10 & $2-1-5$ & 1.28 & 1.65 & 28.91 \\
11 & $2-1-5$ & 1.36 & 1.57 & 15.44 \\
12 & $2-1-5$ & 1.64 & 1.70 & 3.66 \\
\hline \hline
\end{tabular}

the electron flow when compared to spins being parallel to it. SOC thus seems to overcome the effect of back-scattering induced by the domain wall. Other configurations, however, show the expected behavior where the domain wall reduces the conductance. The high sensitivity of the valence $d$ orbitals to the detailed geometry of the contacts makes it difficult to predict in which way the conductance will deviate in going from a saturated to a NC configuration.

Finally, our calculations confirm that the average DWMR due to noncollinear spins is low when the DWs straddle the minimum cross section. However, we obtain maximum individual values in agreement with those measured experimentally (no higher than $50 \%$ in the best-case scenario). The signs of the DWMR can be in either direction, resulting in an average largest DWMR of $\sim 2 \%$, with standard deviation $( \pm \sim 10 \%)$, in this work (see Tables I and II). This behavior resembles the way the Kondo resonance of ferromagnetic $3 d$ transition metal nanocontacts manifests either as a spike or a sharp dip in conductance at zero bias, being very sensitive to the exact atomic arrangement at the constriction [30]. Hence, although DWMR can contribute to broadening conductance peaks, domain walls cannot really shift the mean of the peaks to different values.

\section{B. Experimental results}

As we mentioned in the Introduction, the most widely used statistical approach to analyze data from electronic transport experiments is the histogram of conductance [75,76], which is constructed from thousands of accumulated traces recorded under the same experimental conditions. To build each normalized histogram we have used 5000 traces and 1000 bins in order to cover a range of conductance from $0.01 G_{0}$ to $6 G_{0}$. The test sample that we report here exhibits the two differently shaped normalized histograms, as shown in Fig. 3(a). The orange and gray histograms are denoted "1-peak" and "2-peak," respectively. The unique method that was followed, in order to obtain 1-peak or 2-peak histograms, involved crashing the two tips of the break junction into each other until the measured conductance exceeded $\sim 10 G_{0}$, although this procedure did not always guarantee that the 2-peak histogram would emerge. To shed light on these ambiguous histograms, we have decided to delve into a statistical study of experimental data to clarify whether there is any correlation between the structural behavior of atomic contacts of the same type, i.e., type 1-peak or 2-peak.

In Fig. 3(b), we show six horizontally offset rupture traces for nickel at $4.2 \mathrm{~K}$. Gray and orange traces have been selected from the ensembles of traces that compose the 2-peak and 1-peak histograms, respectively. This figure shows a horizontal yellow band between $1 G_{0}$ and $2 G_{0}$ and a dashed black line at $1.5 G_{0}$ to facilitate comparisons between conductance plateaus that contribute to the peaks in the histogram above or below the line. Usually, gray traces do not register any intermediate counts in the range $\sim 0.1 G_{0}$ to $\sim 1 G_{0}$, in other words, they show abrupt jumps in the conductance between last atomic contact and rupture, also known as "jump out-ofcontact" [46,74]. Conversely, one of the orange traces exhibits a smooth transition between the last contact and rupture. In fact, this absence of jump out-of-contact is evident in the 1-peak histogram as more counts in the range of $\sim 0.1 G_{0}$ to $\sim 1 G_{0}$. The fact that the traces manifest the jump-out-ofcontact phenomenon in some experiments while in others they do not indicates different mechanical behavior during contact rupture. In order to further shed light on the behavior of nickel nanocontacts, we have done a statistical study based on two-dimensional (conductance-displacement) histograms. ${ }^{1}$ The analysis performed in Fig. 3(c) shows that the 2-peak histogram exhibits anticorrelation, which means that the onset of the abrupt conductance fall typically takes place either at $\sim 1.6 G_{0}$ or at $\sim 1.2 G_{0}$, but never occurs together (i.e., one after the other) in the same trace. Anticorrelation between bins reflects the existence of jumps in the conductance. Usually, the jumps in a given trace arise from atomic rearrangements within the contact. Therefore, if the anticorrelation is not an isolated occurrence and appears as an area within Fig. 3(c), it means that similar jumps occur in all the traces. In other words, we are essentially reproducing the same atomic configuration. On the other hand, in Fig. 3(d), which corresponds to the 1-peak histogram, completely different behavior is observed: a large number of traces exhibit a high degree of correlation in certain ranges, which means that the onset of a fall in conductance is not always located within the same range of conductance values and, moreover, only one type of plateau, centered on $\sim 1.6 G_{0}$, occurs before rupture.

\section{Comparison of the simulated and experimental results}

The experimental conductance histograms of $\mathrm{Ni}$ can exhibit either two sharp peaks, centered on $\sim 1.2 G_{0}$ and $\sim 1.6 G_{0}$, or a single broader peak centered on $\sim 1.6 G_{0}$. Our results, collected in Tables I and II, suggest that the experimental

\footnotetext{
${ }^{1}$ See the cross-correlation histograms in Figs. 3(c) and 3(d) of the main text, as well as the Supplemental Material [71] for a more in-depth analysis of the features of the rupture traces used to construct the histograms, including a more detailed statistical analysis via conductance-displacement histograms.
} 

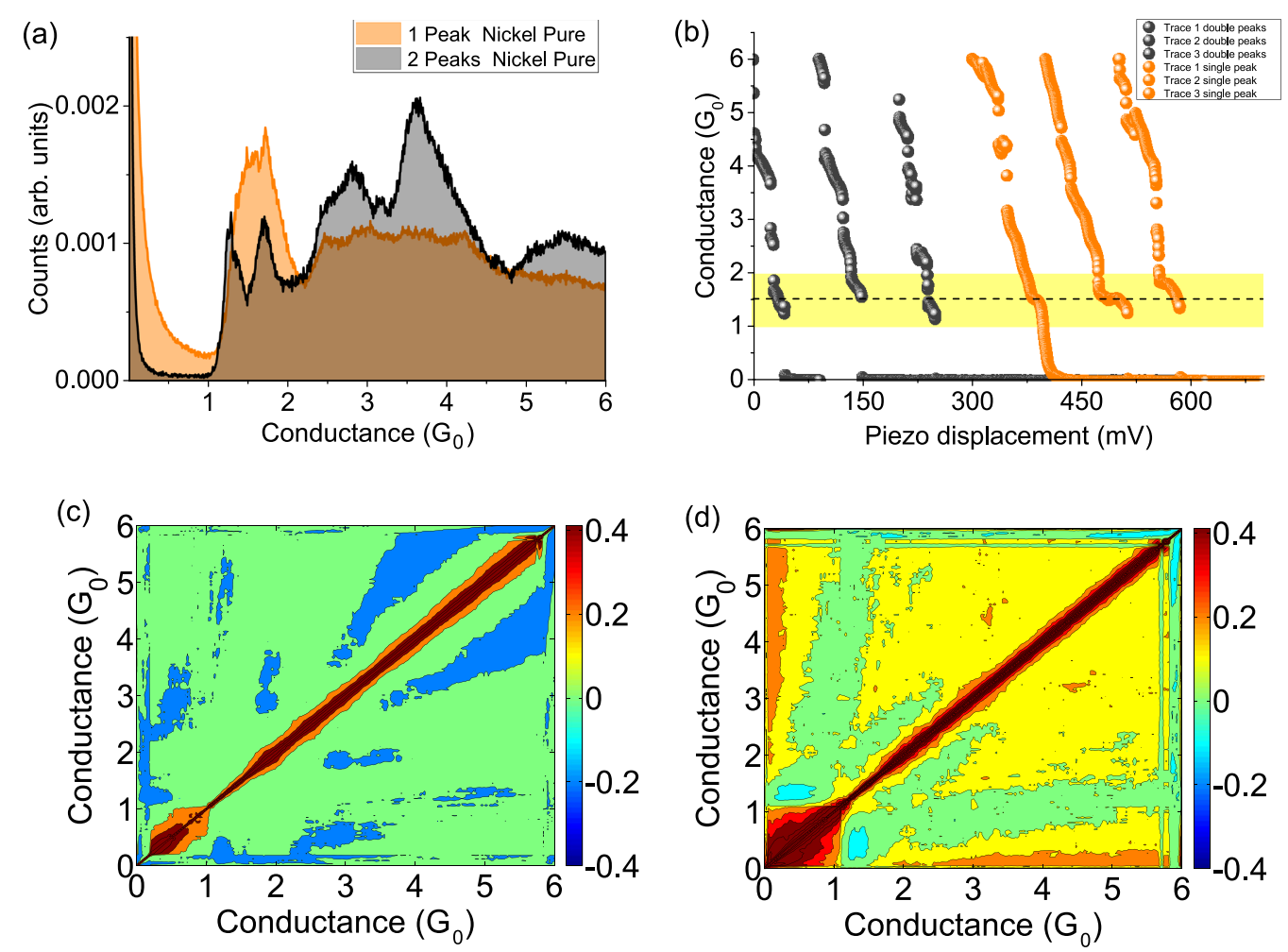

FIG. 3. (a) Conductance histograms constructed from rupture traces for nickel. (b) Traces of rupture for nickel at $4.2 \mathrm{~K}$. The horizontal yellow band highlights values between $1 G_{0}$ to $2 G_{0}$, and a dashed line at around $1.5 G_{0}$ helps to visualize whether the plateau of last contact is above or below this central value. In both (a) and (b) orange corresponds to the traces and histogram whose main feature is " 1 -peak" while gray corresponds to the traces and histogram with "2-peaks." Both (c) and (d) show the results from a statistical analysis known as a two-dimensional cross-correlation histogram [32]. (c) Corresponds to the case in which traces give rise to a 2-peak histogram and (d) to a histogram that exhibits a single peak centered at $1.6 G_{0}$.

conductance histograms of Ni which exhibit the two sharp peaks, as opposed to those exhibiting a single broader peak, can be attributed to highly reproducible structures that form as the contacts thin to the point of rupture, along different crystallographic axes, during cyclic loading. The averaged results of our electronic transport calculations, classified by last contact type (monomer, dimer, or other), are presented in Table III along with their associated uncertainties. The values in Table III are also shown as transparent color-coded strips in Fig. 4(a), i.e., as overlays on the experimental histograms from Fig. 3. These results were calculated from 25 last-contact snapshot configurations in which the majority $(\sim 60 \%)$ of the minimum cross sections were monomers. We thus find that cyclic loading of both (001)- and (111)-oriented nickel nanocontacts favor the formation of monomers [see Fig. 4(b)] after the contacts have been mechanically annealed $[51,74]$.

TABLE III. Influence of the spin collinearity in the calculations of conductance ( $G_{0}$ units) for the structures classified as monomer, dimer, and others of Tables I and II.

\begin{tabular}{lccc}
\hline \hline Collinear & $\begin{array}{c}\text { Dimer } \\
\left(G_{0}\right)\end{array}$ & $\begin{array}{c}\text { Monomer } \\
\left(G_{0}\right)\end{array}$ & $\begin{array}{c}\text { Other } \\
\left(G_{0}\right)\end{array}$ \\
\hline Yes & $1.3 \pm 0.3$ & $1.7 \pm 0.3$ & $2.4 \pm 0.2$ \\
No & $1.2 \pm 0.3$ & $1.8 \pm 0.2$ & $2.3 \pm 0.3$ \\
\hline \hline
\end{tabular}

Notice that the average conductance of (111)-oriented $\mathrm{Ni}$ nanocontacts in Fig. 4(b) is clearly lower than their (001)-oriented counterparts. Under cyclic loading, the EAM potential used in this work favors the formation of longer and narrower stable last-contact structures in the case of (111)-oriented nanocontacts, i.e., similar to the pentagonal wires observed in Ref. [33]. Evidently, longer and narrower nanocontacts give rise to lower conductance values on average at last contact. However, in contrast to Ref. [33], where pentagonal wires formed preferentially in (001)- and (110)oriented $\mathrm{Ni}$ nanocontacts, the cyclic loading performed in our SLD simulations favors the formation of wider and flatter pyramid-shaped tips in the (001) orientation. The reason for this is that (111)-oriented facets are formed on the exposed sides of the (001)-oriented tips during cyclic loading, which, for fcc metals in general, are favored in terms of energy over exposed surface layers of other crystallographic orientations [70].

Moreover, the fact that the average conductance value reported in this work for the (111)-oriented nanocontacts is lower than their (001)-oriented counterparts [see Fig. 4(b)], as well as the small value $(\sim 2 \%)$ of the average DWMR, suggests that the first sharp peak in the gray conductance histogram in Fig. 3(a) can be assigned to (111)-oriented Ni nanocontacts. Then, it is important to mention that the magnetic easy axis of bulk nickel is oriented along the (111) direction and is 20 times more resistant to deviation at $5 \mathrm{~K}$ 


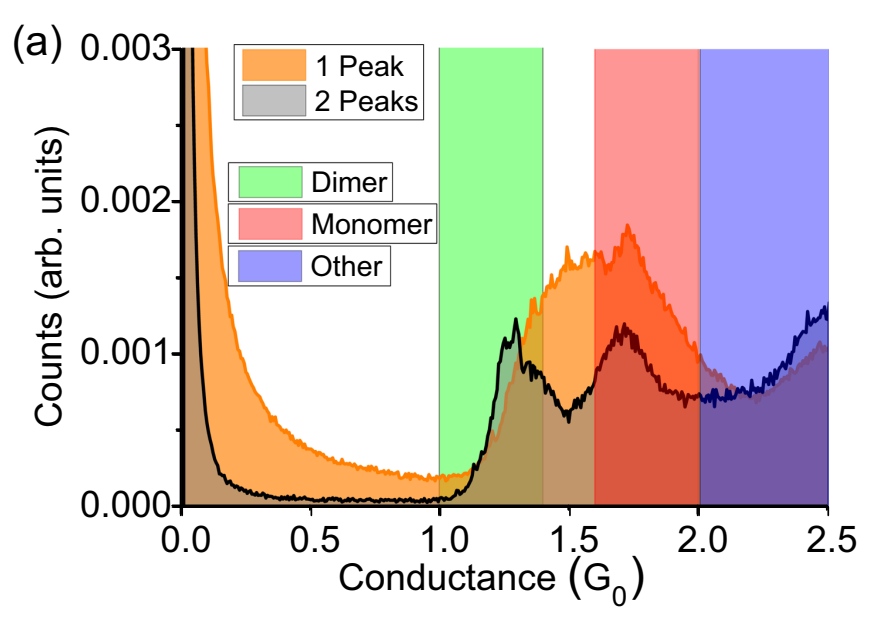

(b)

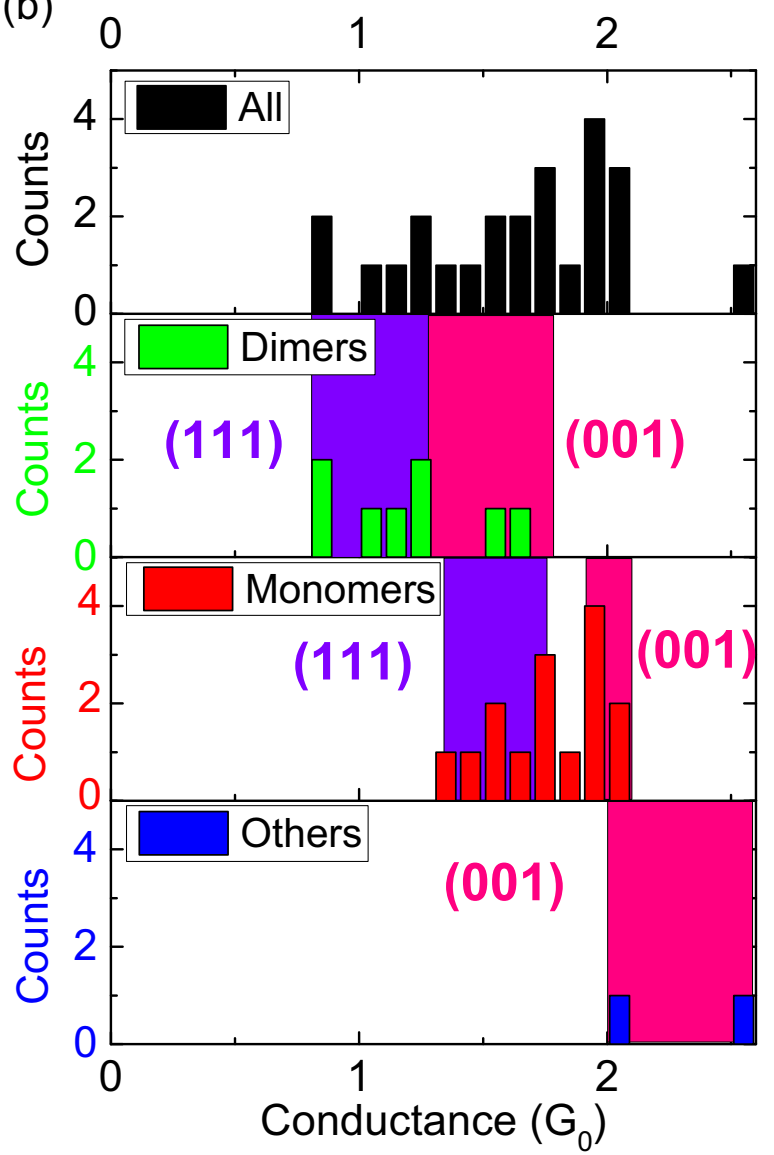

FIG. 4. (a) Ranges of conductance values, from the first row of Table III, corresponding to the NC spin configurations, calculated for dimers (green overlay), monomers (red overlay), and more complex (blue overlay) SLD last-contact structures, compared with the experimental histograms from Fig. 3. (b) Histograms of the calculated conductance, also broken down by contact type: dimer, monomer, and more complex. Purple and pink shading represent the parts of the calculated histograms corresponding to the conductance values of (111)- and (001)-oriented nanocontacts, respectively.

than at room temperature [77]. Therefore, unlike our SLD simulations in which $C_{2}$ was deliberately made negative to favor the formation of transverse noncollinear DWs, in reality, the spins will not rotate to form domain walls that are perpendicular to the (111) crystallographic axis, unless they are coerced by a sufficiently strong external magnetic field acting perpendicularly to the nanocontact. This suggests that the true DWMR of (111)-oriented nancontacts will actually be much lower than the individual values we have calculated in our work.

In the (001) orientation, on the other hand, the spins will be far more likely to point in a direction that is at an angle to the long axis of the nanocontacts. This fact makes provision for a greater variety of possible spin textures in the atomic layers on either side of the minimum cross section. An easy way to visualize this is to twist, about the $z$ axis, the two opposing (001) "pyramids." Since the spins in either pyramid can point in $( \pm 1 \pm 1 \pm 1)$, at an angle to the (001) direction, the spins will not look the same as the two ends are rotated relative to each other about their common axis. In a (111)oriented nanocontact, on the other hand, this operation will essentially leave the spins looking the same since they are overwhelmingly aligned along (111) at $4.2 \mathrm{~K}$. Therefore, the lower symmetry of the spin orientations in $\mathrm{Ni}(001)$ contacts can explain the broader first peak in the 1-peak histogram because they can give rise to greater scattering of electrons impinging upon the minimum cross section. Conversely, we can expect the 2-peak histogram to have sharper peaks because the spins will be far less likely to deviate from the magnetic easy axis in a $\mathrm{Ni}(111)$ nanocontact.

\section{CONCLUSIONS}

In this work, we have presented a combined model of spin-lattice dynamics simulations and electronic transport calculations with post-self-consistent-field corrections for spin-orbit coupling and noncollinear spins. In our combined model we studied and simulated nickel nanocontacts with (001) and (111) crystallographic orientations. From these simulations we extracted the last contact structures to calculate and compare the SOC corrected conductance for fully saturated and noncollinear spin textures. The domainwall magnetoresistance of each structure was then calculated. Based on our results we can claim that the DWMR obtained for atomic-sized contacts made of $\mathrm{Ni}$ is an expected small overall average value of $\sim 2 \%( \pm \sim 10 \%)$.

To validate our refined methodology of SLD simulations and electronic transport calculations we have analyzed experimental traces of conductance of nickel. Our statistical analyses show that there are two histograms for the same material, and that the histogram with two peaks arises from anticorrelated rupture traces. Moreover, we propose that this histogram corresponds to dimers at $\sim 1.2 G_{0}$ and monomers at $\sim 1.6 G_{0}$.

Comparing the experimental results to our combined theoretical model suggests that the first sharp peak in the double-peak histogram arises from very narrow and sharp nanocontacts. Such narrow structures are formed predominantly in our (111)-oriented SLD structures since mechanical annealing produces energetically favored wider and flatter pyramid-shaped structures in (001)-oriented Ni nanocontacts. Our findings suggest that when (111)-oriented Ni nanocontacts, or of any orientations other than (001), are formed in experiments, it is because they represent a local minimum in 
the exposed surface energy. $\mathrm{Ni}(001)$ nanocontacts thus form predominantly because their exposed surfaces, (111) facets, correspond to a global minimum in exposed surface energy. The relative number of monomers and dimers observed in simulations show that the most probable structure at last contact is the monomer, irrespective of crystallographic orientation. Hence, there is a common maximum at $\sim 1.6 G_{0}$ in both types of histogram. Based on all these considerations we rule out, as the underlying explanation for the two types of histograms, the formation of spontaneous domain walls at the constriction of otherwise uniformly magnetized nanocontacts. Rather, the minimization of exposed surface energies and mismatch between the magnetization directions in the bulk electrodes on opposite sides of the constriction provide the best explanation.

In summary, we have developed a general method that can be employed to study any single-element ferromagnetic system in which local coupling between spins and atoms cannot be ignored. In this paper, we have used it to provide an interesting physical interpretation for the electronic transport experiments on nickel.

\section{ACKNOWLEDGMENTS}

W.D. thanks J. Fernández-Rossier for helpful discussions and suggestions to improve the manuscript. This work was supported by the Generalitat Valenciana through Grant No. PROMETEO2017/139. C.S. gratefully acknowledges financial support from the Dean Fellowship of the Weizmann Institute of Science and Generalitat Valenciana (Grant No. CDEIGENT2018/028). O.T. appreciates the support of the Harold Perlman family, and acknowledges funding by a research grant from Dana and Yossie Hollander, the Israel Science Foundation (Grant No. 1089/15), the Minerva Foundation (Grant No. 120865), and The Ministry of Science and Technology of Israel (Grant No. 3-16244). J.J.P. acknowledges financial support from Spanish MINECO through Grants No. FIS2016-80434-P and No. PID2019109539GB-C43, the Fundación Ramón Areces, the María de Maeztu Program for Units of Excellence in R\&D (Grant No. CEX2018-000805-M), the Comunidad Autónoma de Madrid through the Nanomag COST-CM Program (Grant No. S2018/NMT-4321), the European Union Seventh Framework Programme under Grant Agreement No. 604391 Graphene Flagship, the Centro de Computación Científica of the Universidad Autónoma de Madrid and the computer resources at MareNostrum and the technical support provided by the Barcelona Supercomputing Center (Grant No. FI2019-2-0007). The SLD and DFT calculations in this paper were performed on the high-performance computing facilities of the University of Alicante and the University of South Africa.
[1] A. A. Golosov, S. I. Tsonchev, P. Pechukas, and R. A. Friesner, Spin-spin model for two-level system/bath problems: A numerical study, J. Chem. Phys. 111, 9918 (1999).

[2] J. Zázvorka, F. Jakobs, D. Heinze, N. Keil, S. Kromin, S. Jaiswal, K. Litzius, G. Jakob, P. Virnau, D. Pinna, K. Everschor-Sitte, L. Rózsa, A. Donges, U. Nowak, and M. Kläui, Thermal skyrmion diffusion used in a reshuffler device, Nat. Nanotechnol. 14, 658 (2019).

[3] D. Perera, Atomistic simulations of magnetic models with coupled translational and spin degrees of freedom, Ph.D. thesis, University of Georgia, 2015.

[4] D. Perera, M. Eisenbach, D. M. Nicholson, G. M. Stocks, and D. P. Landau, Reinventing atomistic magnetic simulations with spin-orbit coupling, Phys. Rev. B 93, 060402(R) (2016).

[5] M. Aßmann and U. Nowak, Spin-lattice relaxation beyond Gilbert damping, J. Magn. Magn. Mater. 469, 217 (2018).

[6] J. M. Soler, E. Artacho, J. D. Gale, A. García, J. Junquera, P. Ordejón, and D. Sánchez-Portal, The SIESTA method for ab initio order-N materials simulation, J. Phys.: Condens. Matter 14, 2745 (2002).

[7] A. Smogunov, A. Dal Corso, and E. Tosatti, Selective d-state conduction blocking in nickel nanocontacts, Surf. Sci. 507-510, 609 (2002).

[8] A. Bagrets, N. Papanikolaou, and I. Mertig, Magnetoresistance of atomic-sized contacts: An ab initio study, Phys. Rev. B 70, 064410 (2004).

[9] A. Delin and E. Tosatti, The electronic structure of $4 \mathrm{~d}$ transition-metal monatomic wires, J. Phys.: Condens. Matter 16, 8061 (2004).
[10] A. Smogunov, A. Dal Corso, and E. Tosatti, Ballistic conductance of magnetic $\mathrm{Co}$ and $\mathrm{Ni}$ nanowires with ultrasoft pseudopotentials, Phys. Rev. B 70, 045417 (2004).

[11] J. Velev, R. F. Sabirianov, S. S. Jaswal, and E. Y. Tsymbal, Ballistic Anisotropic Magnetoresistance, Phys. Rev. Lett. 94, 127203 (2005).

[12] A. Smogunov, A. Dal Corso, and E. Tosatti, Ballistic conductance and magnetism in short tip suspended Ni nanowires, Phys. Rev. B 73, 075418 (2006).

[13] F. Pauly, J. K. Viljas, U. Huniar, M. Häfner, S. Wohlthat, M. Bürkle, J. C. Cuevas, and G. Schön, Cluster-based densityfunctional approach to quantum transport through molecular and atomic contacts, New J. Phys. 10, 125019 (2008).

[14] S. Achilles, M. Czerner, and I. Mertig, Tailoring magnetoresistance through rotating Ni particles, Phys. Rev. B 84, 054418 (2011).

[15] B. Hardrat, F. Freimuth, S. Heinze, and Y. Mokrousov, Conductance fingerprints of noncollinear magnetic states in single-atom contacts: A first-principles Wannier-functions study, Phys. Rev. B 86, 165449 (2012).

[16] J. Ferrer, C. J. Lambert, V. M. García-Suárez, D. Z. Manrique, D. Visontai, L. Oroszlany, R. Rodríguez-Ferradás, I. Grace, S. W. D. Bailey, K. Gillemot, H. Sadeghi, and L. A. Algharagholy, GOLLUM: A next-generation simulation tool for electron, thermal and spin transport, New J. Phys. 16, 093029 (2014).

[17] T. Ozaki, K. Nishio, and H. Kino, Efficient implementation of the nonequilibrium Green function method for electronic transport calculations, Phys. Rev. B 81, 035116 (2010). 
[18] T. Ozaki, H. Kino, J. Yu, M. Han, M. Ohfuchi, F. Ishii, K. Sawada, Y. Kubota, Y. Mizuta, T. Ohwaki, T. Duy, H. Weng, Y. Shiihara, M. Toyoda, Y. Okuno, R. Perez, P. Bell, M. Ellner, Y. Xiao, A. Ito et al., OpenMX (Open source package for Material eXplorer) ver. 3.8, http://www.openmx-square.org/

[19] D. Jacob, J. Fernández-Rossier, and J. J. Palacios, Magnetic and orbital blocking in Ni nanocontacts, Phys. Rev. B 71, 220403(R) (2005).

[20] D. Jacob and J. J. Palacios, Orbital eigenchannel analysis for abinitio quantum transport calculations, Phys. Rev. B 73, 075429 (2006).

[21] D. Jacob, J. Fernández-Rossier, and J. J. Palacios, Anisotropic magnetoresistance in nanocontacts, Phys. Rev. B 77, 165412 (2008).

[22] R. Requist, P. P. Baruselli, A. Smogunov, M. Fabrizio, S. Modesti, and E. Tosatti, Metallic, magnetic and molecular nanocontacts, Nat. Nanotechnol. 11, 499 (2016).

[23] S. Pakdel, M. Pourfath, and J. J. Palacios, An implementation of spin-orbit coupling for band structure calculations with gaussian basis sets: Two-dimensional topological crystals of $\mathrm{Sb}$ and Bi, Beilstein J. Nanotechnol. 9, 1015 (2018).

[24] C. Barreteau, D. Spanjaard, and M.-C. Desjonquères, An efficient magnetic tight-binding method for transition metals and alloys, C. R. Phys. 17, 406 (2016).

[25] C. Michael, Y. Bogdan, and M. Ingrid, The role of noncollinear magnetic order and magnetic anisotropy for the transport properties through nanowires, Phys. Status Solidi B 247, 2594 (2010).

[26] M. R. Calvo, M. J. Caturla, D. Jacob, C. Untiedt, and J. J. Palacios, Mechanical, electrical, and magnetic properties of ni nanocontacts, IEEE Trans. Nanotechnol. 7, 165 (2008).

[27] M. Kumar, K. K. V. Sethu, and J. M. van Ruitenbeek, Moleculeassisted ferromagnetic atomic chain formation manohar, Phys. Rev. B 91, 245404 (2015).

[28] R. Vardimon, M. Matt, P. Nielaba, J. C. Cuevas, and O. Tal, Orbital origin of the electrical conduction in ferromagnetic atomic-size contacts: Insights from shot noise measurements and theoretical simulations, Phys. Rev. B 93, 085439 (2016).

[29] C. Untiedt, D. M. T. Dekker, D. Djukic, and J. M. van Ruitenbeek, Absence of magnetically induced fractional quantization in atomic contacts, Phys. Rev. B 69, 081401(R) (2004).

[30] M. R. Calvo, J. Fernández-Rossier, J. J. Palacios, D. Jacob, D. Natelson, and C. Untiedt, The Kondo effect in ferromagnetic atomic contacts., Nature (London) 458, 1150 (2009).

[31] A. Yanson, Atomic chains and electronic shells: Quantum mechanisms for the formation of nanowires, Ph.D. thesis, Universiteit Leiden, 2001.

[32] A. Halbritter, P. Makk, S. Mackowiak, S. Csonka, M. Wawrzyniak, and J. Martinek, Regular Atomic Narrowing of $\mathrm{Ni}, \mathrm{Fe}$, and V Nanowires Resolved by Two-Dimensional Correlation Analysis, Phys. Rev. Lett. 105, 266805 (2010).

[33] P. García-Mochales, R. Paredes, S. Peláez, and P. A. Serena, Statistical analysis of the breaking processes of Ni nanowires, Nanotechnology 19, 225704 (2008).

[34] A. Fert, N. Reyren, and V. Cros, Magnetic skyrmions: Advances in physics and potential applications, Nat. Rev. Mater. 2, 17031 (2017).

[35] J. M. Caridad, D. Mccloskey, J. F. Donegan, and V. Krstić, Con- trollable growth of metallic nano-helices at room temperature conditions, Appl. Phys. Lett. 105, 233114 (2014).

[36] D. B. Reeves and J. B. Weaver, Approaches for modeling magnetic nanoparticle dynamics, CRC Crit. Rev. Biomed. Eng 42, 85 (2014).

[37] A. Einstein and W. J. de Haas, Experimental proof of the existence of Ampère's molecular currents, in Proceedings of the Royal Netherlands Academy of Arts and Sciences (KNAW, 1915), Vol. 8 I, p. 696.

[38] S. J. Barnett, Magnetization by rotation, Phys. Rev. 6, 239 (1915).

[39] B. D. Cullity and C. D. Graham, Introduction to Magnetic Materials, 2nd ed. (Wiley, Hoboken, NJ, 2009).

[40] P.-W. Ma, C. H. Woo, and S. L. Dudarev, Large-scale simulation of the spin-lattice dynamics in ferromagnetic iron, Phys. Rev. B 78, 024434 (2008).

[41] D. Beaujouan, P. Thibaudeau, and C. Barreteau, Anisotropic magnetic molecular dynamics of cobalt nanowires, Phys. Rev. B 86, 174409 (2012).

[42] P.-W. Ma, S. L. Dudarev, and C. H. Woo, SPILADY: A parallel CPU and GPU code for spin-lattice magnetic molecular dynamics simulations, Comput. Phys. Commun. 207, 350 (2016).

[43] M. S. Daw and M. I. Baskes, Semiempirical, Quantum Mechanical Calculation of Hydrogen Embrittlement in Metals, Phys. Rev. Lett. 50, 1285 (1983).

[44] X. Zhou, H. Wadley, R. Johnson, D. Larson, N. Tabat, A. Cerezo, A. Petford-Long, G. Smith, P. Clifton, R. Martens, and T. Kelly, Atomic scale structure of sputtered metal multilayers, Acta Mater. 49, 4005 (2001).

[45] H. Wadley, X. Zhou, R. Johnson, and M. Neurock, Mechanisms, models and methods of vapor deposition, Prog. Mater Sci. 46, 329 (2001).

[46] M. A. Fernández, C. Sabater, W. Dednam, J. J. Palacios, M. R. Calvo, C. Untiedt, and M. J. Caturla, Dynamic bonding of metallic nanocontacts: Insights from experiments and atomistic simulations, Phys. Rev. B 93, 085437 (2016).

[47] C. Sabater, W. Dednam, M. R. Calvo, M. A. Fernández, C. Untiedt, and M. J. Caturla, Role of first-neighbor geometry in the electronic and mechanical properties of atomic contacts, Phys. Rev. B 97, 075418 (2018).

[48] W. Dednam, C. Sabater, M. R. Calvo, C. Untiedt, J. J. Palacios, A. E. Botha, and M. J. Caturla, Directional bonding explains the high conductance of atomic contacts in bcc metals, Phys. Rev. B 101, 165417 (2020).

[49] M. Eisenbach, D. Perera, D. P. Landau, D. M. Nicholson, J. Yin, and G. Brown, Magnetic materials at finite temperatures: Thermodynamics and combined spin and molecular dynamics derived from first principles calculations, J. Phys.: Conf. Ser. 640, 012019 (2015).

[50] A. M. Bratkovsky, A. P. Sutton, and T. N. Todorov, Conditions for conductance quantization in realistic models of atomic-scale metallic contacts, Phys. Rev. B 52, 5036 (1995).

[51] C. Sabater, C. Untiedt, J. J. Palacios, and M. J. Caturla, Mechanical Annealing of Metallic Electrodes at the Atomic Scale, Phys. Rev. Lett. 108, 205502 (2012).

[52] W. Dednam, Atomistic simulations of competing influences on electron transport across metal nanocontacts, Ph.D. thesis, University of Alicante/University of South Africa, 2019.

[53] P.-W. Ma (private communication). 
[54] M. Pajda, J. Kudrnovský, I. Turek, V. Drchal, and P. Bruno, Ab initio calculations of exchange interactions, spin-wave stiffness constants, and Curie temperatures of $\mathrm{Fe}, \mathrm{Co}$, and Ni, Phys. Rev. B 64, 174402 (2001).

[55] A. Von Bieren, A. K. Patra, S. Krzyk, J. Rhensius, R. M. Reeve, L. J. Heyderman, R. Hoffmann-Vogel, and M. Kläui, DomainWall Induced Large Magnetoresistance Effects at Zero Applied Field in Ballistic Nanocontacts, Phys. Rev. Lett. 110, 067203 (2013).

[56] N. Agraït, J. G. Rodrigo, and S. Vieira, Conductance steps and quantization in atomic-size contacts, Phys. Rev. B 47, 12345 (1993).

[57] J. J. Palacios, A. J. Pérez-Jiménez, E. Louis, and J. A. Vergés, Fullerene-based molecular nanobridges: A first-principles study, Phys. Rev. B 64, 115411 (2001).

[58] J. J. Palacios, A. J. Pérez-Jiménez, E. Louis, E. SanFabián, and J. A. Vergés, First-principles approach to electrical transport in atomic-scale nanostructures, Phys. Rev. B 66, 035322 (2002).

[59] E. Louis, J. A. Vergés, J. J. Palacios, A. J. Pérez-Jiménez, and E. SanFabián, Implementing the keldysh formalism into $\mathrm{ab}$ initio methods for the calculation of quantum transport: Application to metallic nanocontacts, Phys. Rev. B 67, 155321 (2003).

[60] J. J. Palacios et al., Computer code ANT.Gaussian, with SOC corrections, available from https://github.com/juanjosepalacios/ ANT.Gaussian.

[61] M. J. Frisch, G. W. Trucks, H. B. Schlegel, G. E. Scuseria, M. A. Robb, J. R. Cheeseman, G. Scalmani, V. Barone, G. A. Petersson, H. Nakatsuji, X. Li, M. Caricato, A. Marenich, J. Bloino, B. G. Janesko, R. Gomperts, B. Mennucci, H. P. Hratchian, J. V. Ortiz, A. F. Izmaylov et al., computer code GAUSSIAN09, Revision C.01 (Gaussian, Inc., Wallingford, CT, 2020).

[62] P. Giannozzi, S. Baroni, N. Bonini, M. Calandra, R. Car, C. Cavazzoni, D. Ceresoli, G. L. Chiarotti, M. Cococcioni, I. Dabo, A. D. Corso, S. de Gironcoli, S. Fabris, G. Fratesi, R. Gebauer, U. Gerstmann, C. Gougoussis, A. Kokalj, M. Lazzeri, L. Martin-Samos et al., Quantum espresso: A modular and open-source software project for quantum simulations of materials, J. Phys.: Condens. Matter 21, 395502 (2009).

[63] R. Dovesi, R. Orlando, A. Erba, C. M. Zicovich-Wilson, B. Civalleri, S. Casassa, L. Maschio, M. Ferrabone, M. De La Pierre, P. D'Arco, Y. Noël, M. Causà, M. Rérat, and B. Kirtman, Crystal14: A program for the ab initio investigation of crystalline solids, Int. J. Quantum Chem. 114, 1287 (2014).

[64] R. M. Reeve, A. Loescher, H. Kazemi, B. Dupé, M.-A. Mawass,
T. Winkler, D. Schönke, J. Miao, K. Litzius, N. Sedlmayr, I. Schneider, J. Sinova, S. Eggert, and M. Kläui, Scaling of intrinsic domain wall magnetoresistance with confinement in electromigrated nanocontacts, Phys. Rev. B 99, 214437 (2019).

[65] T. Schena, Tight-binding treatment of complex magnetic structures in low-dimensional systems, Master's thesis, Aachen University, Germany, 2010.

[66] J. I. Pascual, J. Méndez, J. Gómez-Herrero, A. M. Baró, N. García, and V. T. Binh, Quantum Contact in Gold Nanostructures by Scanning Tunneling Microscopy, Phys. Rev. Lett. 71, 1852 (1993).

[67] C. Muller, J. van Ruitenbeek, and L. de Jongh, Experimental observation of the transition from weak link to tunnel junction, Phys. C (Amsterdam) 191, 485 (1992).

[68] C. J. Muller, J. M. van Ruitenbeek, and L. J. de Jongh, Conductance and Supercurrent Discontinuities in Atomic-Scale Metallic Constrictions of Variable Width, Phys. Rev. Lett. 69, 140 (1992).

[69] N. Agraït, A. L. Yeyati, and J. M. Van Ruitenbeek, Quantum properties of atomic-sized conductors, Phys. Rep. 377, 81 (2003).

[70] W. Dednam, C. Sabater, M. A. Fernández, C. Untiedt, J. J. Palacios, and M. J. Caturla, Modeling contact formation between atomic-sized gold tips via molecular dynamics, J. Phys.: Conf. Ser. 574, 012045 (2015).

[71] See Supplemental Material at http://link.aps.org/supplemental/ 10.1103/PhysRevB.102.245415 where the full process of rupture and formation of the spins in our simulated nanocontacts are shown in two movies, named movie001.avi and movie111.avi.

[72] K. Doll, Density functional study of Ni bulk, surfaces and the adsorbate systems $\mathrm{Ni}(111)(3 \times 3) \mathrm{R} 30^{\circ}-\mathrm{Cl}$, and $\mathrm{Ni}(111)(2 \times$ 2)-K, Surf. Sci. 544, 103 (2003).

[73] F. Körmann, P. W. Ma, S. L. Dudarev, and J. Neugebauer, Impact of magnetic fluctuations on lattice excitations in fcc nickel, J. Phys.: Condens. Matter 28, 076002 (2016).

[74] C. Untiedt, M. J. Caturla, M. R. Calvo, J. J. Palacios, R. C. Segers, and J. M. van Ruitenbeek, Formation of a Metallic Contact: Jump to Contact Revisited, Phys. Rev. Lett. 98, 206801 (2007).

[75] J. M. Krans, C. J. Muller, I. K. Yanson, T. C. M. Govaert, R. Hesper, and J. M. van Ruitenbeek, One-atom point contacts, Phys. Rev. B 48, 14721 (1993).

[76] J. Krans, J. van Ruitenbeek, and L. de Jongh, Atomic structure and quantized conductance in metal point contacts, Phys. B (Amsterdam) 218, 228 (1996).

[77] D. I. Bower, The magnetostriction coefficients of nickel, Proc. R. Soc. London, Ser. A 326, 87 (1971). 